Revista Internacional de

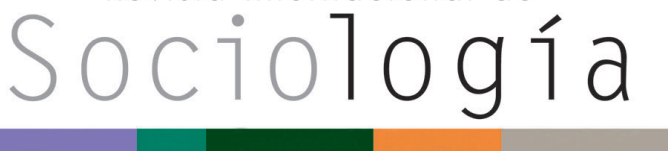

\section{ENCARCELAMIENTO, LAZOS FAMILIARES Y REINCIDENCIA. Explorando los límites del familismo}

Joel Martí

Universitat Autònoma de Barcelona, España.

Joel.Marti@uab.cat

JosÉ CID

Universitat Autònoma de Barcelona, España.

Josep.Cid@uab.es

Como citar este artículo / Citation: "Encarcelamiento, lazos familiares y reincidencia. Explorando los límites del familismo", Revista Internacional de Sociología, 73 (1), doi: http://dx.doi.org/103989/ris.2013.02.04

\section{RESUMEN}

Este artículo analiza los efectos en la reincidencia de los contactos familiares durante el encarcelamiento y explora los mecanismos explicativos que vinculan ambos factores, en un contexto en el que las familias ejercen un rol central en la provisión de bienestar. Para ello se plantea un diseño mixto, en dos olas, centrado en el estudio de hombres condenados a prisión por delincuencia común en la provincia de Barcelona. El análisis muestra que tanto los lazos familiares más fuertes (parejas y padres) como los más débiles (con otros familiares) tienen un efecto relevante en el proceso de desistimiento, aunque la forma en que operan es distinta (vínculo en el primer caso, apoyo en el segundo). Sin embargo, los resultados muestran también los límites del vínculo familiar cuando no va acompañado de los recursos materiales necesarios para conducir con éxito la transición postpenitenciaria. El análisis cualitativo permite profundizar en los factores explicativos e introducir varios matices a estas conclusiones mediante la observación de casos no confirmatorios.

\section{Palabras Clave}

Delincuencia; Desistimiento; Familia; Métodos mixtos; Prisión.
Revista Internacional de Sociología RIS

DOI: http://dx.doi.org/10.3989/ris.2013.02.04

vol. 73 (1), e002, enero-abril, 2015, ISSN-L: 0034-9712

\section{IMPRISONMENT, FAMILY TIES AND RECIDIVISM. Exploring the limits of familism}

Copyright: @2015 CSIC. Este artículo de acceso abierto distribuido bajo los terminos de la licencia Creative Commons Attribution-Non Commercial (by-cn) Spain 3.0.

Recibido: 04/02/2013. Aceptado: 19/12/2013

\section{Abstract}

This paper analyzes the effects of family contacts during incarceration on recidivism and explores the mechanisms that relate these factors. A two-wave mixed method design is applied on a sample of men given prison sentences for ordinary acquisitive crimes in the province of Barcelona. The analysis shows that both the stronger family ties (with parents and partners) and the weaker ones (with other family members) may have a significant effect on the desistance process, through the mechanism of bonding (in relation to strong ties) or social support (in relation to weak ties). However, the results also show the limitations of bonding when not coupled with the instrumental support needed for a successful reentry. Additionally to quantitative analysis, qualitative analysis has been conducted to detail explanatory mechanisms, taking also into account negative cases.

\section{KEYWORDS}

Delinquency; Desistance; Family; Mixed Methods; Prison. 


\section{INTRODUCCIÓN ${ }^{1}$}

Los lazos familiares han sido largamente señalados en la literatura como factor protector en la transición postpenitenciaria a la vida en libertad. Referencias a ello se encuentran en Ohlin (1951), Glaser (1964), Hairston (1988) y Schroeder, Giordano y Cernkovich (2010). Otros trabajos como los de Duwe y Clark (2013); Cobbina, Huebner y Berg (2012); Mears et al. (2012); Berg y Huebner (2011) y Bales y Mears (2008), han profundizado en el estudio de la relación entre la reincidencia y los lazos familiares durante el encarcelamiento, concluyendo que la presencia e intensidad de dichos lazos está asociada a una reducción y retraso de la reincidencia, si bien los resultados no son siempre coincidentes en identificar qué clase de familiares tienen más relevancia en esta reducción ni cuáles son los mecanismos implicados.

Sin embargo, otras contribuciones señalan que estos contactos pueden tener el efecto contrario. En primer lugar, pueden facilitar la reincidencia si el propio entorno familiar está vinculado a actividades delictivas (Visher y O'Connell 2012). En segundo lugar, puede ser fuente de tensión por su exigencia o presión hacia las personas excarceladas, 0 por las excesivas expectativas de apoyo que estas proyectan hacia sus familiares (Martínez y Abrams 2013; Dixey y Woodall 2012; Bahr et al. 2010). Todo ello en un contexto en el que el encarcelamiento implica presión económica y una vivencia traumática para el entorno familiar y especialmente para las mujeres, que protagonizan el contacto y apoyo (García-Borés et al. 2006; Comfort 2003).

Este trabajo quiere contribuir a la literatura que trata de identificar la influencia que puede tener en la transición postpenitenciaria el mantenimiento de distintos tipos de lazos familiares durante el encarcelamiento y hacerlo en un contexto sur-europeo, en el que las familias -y especialmente las mujeres- asumen un rol central en la provisión de bienestar. Para ello, se centra en el análisis de una muestra de

\footnotetext{
${ }^{1}$ Investigación financiada por el Ministerio de Ciencia e Innovación (DER 2008-05041, "Políticas de reinserción en el ámbito penal"), la Generalitat de Cataluña (SGR-01117 2009) y por el Centre d'Estudis Jurídics i Formació Especialitzada (Ayudas a la Investigación 2010). En la investigación han participado, además de los autores, Aina Ibáñez (coordinadora del trabajo de campo), Eugenia Albani, Anna Meléndez y Ferran Restrepo. Agradecemos a la Dirección General de Servicios Penitenciarios y Rehabilitación del Departamento de Justicia de Cataluña (DGSPR) y, muy especialmente, a Miquel Ángel Esteban, Jefe del Servicio de Rehabilitación, por poner a nuestra disposición la información necesaria para seleccionar y contactar con los participantes. También al conjunto de profesionales de los centros penitenciarios que nos han facilitado el acceso a las personas entrevistadas. Los autores agradecen también los útiles consejos de Marc Ajenjo, Pedro López Roldán y Juanjo Medina en distintas fases del análisis, y a los revisores/as por sus comentarios a una primera versión del artículo. Por último, agradecemos muy especialmente a todas y cada una de las personas entrevistadas la disposición a compartir sus vivencias con nosotros.
}

hombres condenados a prisión en la provincia de Barcelona por delincuencia común orientada al beneficio económico (delitos contra la propiedad y/o tráfico de drogas).

\section{MARCO TEÓRICO}

\section{Explicaciones sobre la relación entre familia y reincidencia}

En la literatura pueden identificarse tres teorías que sustentarían la relación entre familia y (no) reincidencia: la teoría del apoyo social, la teoría del control social informal en el curso de la vida y la teoría de la transformación cognitiva.

La primera explicación proviene de la teoría del apoyo social (Wright, Cullen y Miller 2001; Cullen 1994) y su aportación a la teoría de la tensión (Cullen y Wright 1997). Como indican Mills y Codd (2008), la razón más obvia por la que los familiares pueden ayudar en la transición a la vida en libertad es que son fuente de apoyo. Pueden cubrir las necesidades básicas de vivienda, manutención y cuidado; también pueden ser fuente directa de empleo 0 canal de inserción laboral. Además, la relación social es un recurso en sí mismo en tanto que proveedora de apoyo expresivo (Cullen 1994; Lin 1986). Algunas contribuciones recientes apoyarían este tipo de explicación, especialmente en la vertiente del apoyo instrumental (Mears et al. 2012; Berg y Huebner 2011; La Vigne, Visher y Castro 2004).

Un segundo tipo de explicación deriva de la perspectiva del curso de la vida (life-course theory) (Sampson, Laub y Wimer 2006; Laub y Sampson 2003; Sampson y Laub 1993). Nuevos acontecimientos vitales como la institucionalización de una relación de pareja pueden ejercer de punto de inflexión (turning point) si conllevan que la persona asuma nuevos roles incompatibles con los que le han llevado a la cárcel: los lazos familiares jugarían aquí un papel de vínculo (bonding), disuadiendo a la persona de reincidir si quiere seguir manteniendo dichos lazos. Otros trabajos han señalado que el vínculo puede construirse no solo con nuevas relaciones, sino también fortaleciendo las existentes, cuando estas se han consolidado a lo largo de la condena mediante un contacto regular (Dixey y Woodall 2012).

Una tercera explicación se vincula al interaccionismo simbólico y en particular a las contribuciones de Lemert (1967) sobre el etiquetamiento. Si las acciones tienen su base en las atribuciones que los demás hacen de uno mismo y en la autopercepción que de ello resulta, entonces el desistimiento de la delincuencia debe pasar necesariamente por una transformación de la identidad (Giordano, Cernkovich y Rudolph 2002; Maruna 2001). En este contexto, la teoría de la transformación cognitiva permite abordar el efecto "desetiquetador" que pueden tener las relaciones familiares extrapenitenciarias para contrarrestar los posibles efectos criminógenos de la prisión (Cid 2009; 
García-Borés 2003): facilitando que las personas rompan con el estigma (Calverley 2013) y reforzando la percepción de autoeficacia (Maruna 2001; Hairston 1988).

\section{Contactos con familiares durante el encarcelamiento, cambio cognitivo y reincidencia}

Ante esta diversidad teórica son varios los trabajos que han señalado la relevancia de discernir los mecanismos por los que los contactos con familiares durante el encarcelamiento inciden en la transición postpenitenciaria y las posibles vinculaciones entre las explicaciones anteriores (Mears et al. 2012; Visher y Travis 2003). Sin embargo, buena parte de la investigación realizada en el campo, de tipo cuantitativo y centrada en analizar la asociación estadística entre las visitas de familiares y la reincidencia posterior $^{2}$, no permite clarificar cuáles son estos mecanismos.

Es en este contexto donde las contribuciones realizadas en el marco de la teoría de la transformación cognitiva (Giordano, Cernkovich y Schroeder 2007; Giordano, Cernkovich y Rudolph 2002; Maruna 2001) resultan especialmente pertinentes, al plantear que es la atención a los factores subjetivos lo que permite identificar los mecanismos por los que los lazos familiares inciden sobre el desistimiento. El objeto de este enfoque ya no es la reincidencia como estado final, sino el proceso a través del cual la persona deja de delinquir y, particularmente, el papel que en este proceso juega la percepción sobre la propia identidad y la autoeficacia.

Desde esta perspectiva, lo que se plantea en este artículo es estudiar la relación entre los lazos familiares y la autopercepción en la etapa final de la condena como parte del mecanismo que vincula familia y reincidencia. Algunos de los trabajos sobre desistimiento se centran, justamente, en estudiar las autopercepciones en esta etapa (Visher y O'Connell 2012; Lebel et al. 2008; Burnett 1992). Así, Visher y O'Connell (íbid.) señalan que la autoeficacia al salir de la cárcel está significativamente asociada con el apoyo familiar percibido. Por su parte, Lebel et al. (op. cit.)

\footnotetext{
${ }^{2}$ Aunque en términos generales la investigación -realizada mayoritariamente en países anglosajones- confirma esta relación, a la hora de precisar los efectos específicos de los distintos lazos (parejas, padres, hijos/as, otros familiares), los resultados divergen. Algunas investigaciones señalan como especialmente relevantes los lazos de pareja (Mears et al. 2012; Bales y Mears 2008); otras, los lazos con parientes menos directos (Duwe y Clark 2013; Berg y Huebner 2011). La relación con los padres (refiriéndonos indistintamente a madre y/o padre) parece tener un papel más débil (Berg y Huebner 2011; Bales y Mears 2008) o deja de tenerlo en hombres con mayor historial delictivo (Cobbina, Huebner y Berg 2012). Finalmente, las relaciones con hijos e hijas presentan resultados no coincidentes en la investigación y en algunos casos incluso parecen aumentar la probabilidad de reincidencia (Visher y O'Connell 2012; Savolainen 2009; Bales y Mears 2008; Siennick y Osgood 2008; Wakefield y Uggen 2004).
}

observan cómo la identidad y la percepción de autoeficacia al final de la condena son predictores de la transición postpenitenciaria una vez controladas las previas dificultades económicas, familiares y el consumo drogas.

\section{Contexto de estudio e hipótesis: familismo, crisis económica y desistimiento}

Los trabajos que en nuestro contexto analizan el papel del entorno familiar en el encarcelamiento (por ejemplo, GarcíaBorés et al. 2006) se han centrado más en las consecuencias sobre las familias que sobre las personas encarceladas. Sin embargo, este contexto presenta varias características que lo hacen de especial interés para analizar cómo inciden en el desistimiento delictivo los lazos familiares.

En primer lugar, se sitúa junto con otros países del sur de Europa en lo que la literatura ha denominado el "modelo de bienestar mediterráneo" (Moreno 2001), caracterizado por una centralidad de la familia en la provisión de bienestar material e inmaterial. Esta organización familista conserva en buena medida los roles tradicionales de división sexual del trabajo: mientras que se reserva a los hombres el rol de proveedores dinerarios principales (male breadwinners), son las mujeres quienes, aun participando en el mercado de trabajo, continúan asumiendo el rol de cuidado y atención al resto de miembros del hogar, amortiguando el escaso desarrollo de políticas públicas de bienestar.

En segundo lugar, este papel amortiguador de la familia ha adquirido especial importancia en un contexto de profunda crisis económica: la "vivienda familiar" pasa a ser un recurso de habitabilidad fundamental para todos los miembros con dificultades para emanciparse, y los lazos familiares son un canal básico para transferir rentas en situación de necesidad económica y/o para proporcionar vías de entrada o contacto con la economía informal ante la falta de empleo formal. Con todo, cabe tener en cuenta que, a las dificultades con la que se encuentra la población excarcelada en su reinserción al mercado de trabajo, se añade la vulnerabilidad económica que suele caracterizar su entorno familiar (Rodríguez, Rovira y Larrauri 2013; Alós et al. 2011; García-Borés et al. 2006).

Una tercera característica a considerar es el sensible incremento de la población extranjera en los últimos años. Este colectivo, que ocupa las posiciones más débiles de la estructura social, ha aumentado su presencia hasta representar en Cataluña, en el año 2010, el 16\% de los residentes totales (datos del Padrón de Habitantes) y el $44 \%$ de la población penitenciaria (datos de Idescat, Dirección General de Régimen Penitenciario y Recursos de Cataluña). Si bien se trata en muchos casos de población que ha emigrado a España con su familia o que se ha reagrupado posteriormente con sus familiares más próximos, en otros casos los lazos familiares más fuertes se mantienen en el país de origen. Este fenómeno plantea 
el interés de observar cuál es el papel del entorno familiar cuando el lazo es transnacional.

Finalmente, y con respecto al modelo penitenciario, aunque España comparte con el sistema anglosajón la tendencia hacia un modelo más punitivo, con largas condenas y altas tasas de encarcelamiento, el sistema español mantiene ciertas características que a priori facilitarían un mayor contacto de la persona encarcelada con sus redes sociales: sistema de permisos, mayor uso del régimen abierto y posibilidad (aunque de aplicación discrecional) de transición escalonada a la vida en libertad (García-España y Díez Ripollés 2012; Cid 2005; Petersilia 2003). Asimismo, el Reglamento Penitenciario español contempla varias formas de comunicación de las personas internas con sus contactos exteriores (presenciales, telefónicas y postales), aunque restringidas en horarios, tiempos, autonomía e intimidad y con especiales dificultades en distancias internacionales (Castillo y Ruiz 2010; García-Borés et al. 2006).

En síntesis, se trata de un contexto en el que parece esperable que el entorno familiar juegue un rol importante en el proceso de desistimiento. En este sentido, y a partir del marco presentado, el análisis está orientado a contrastar hipótesis relativas a la relación entre el contacto familiar durante el encarcelamiento, la autopercepción al finalizar la condena y la posterior reincidencia o desistimiento delictivo. Particularmente, la primera hipótesis que planteamos es que el contacto familiar durante el encarcelamiento tendrá un efecto relevante en la autopercepción desistente al final de la condena. Además, el análisis persigue discernir (a) qué tipos de contactos familiares son más relevantes; (b) qué mecanismos explican la relación entre contactos familiares y autopercepción desistente. Una segunda hipótesis, basada en la teoría de la transformación cognitiva, apunta a que la autopercepción tendrá un papel fundamental en el desistimiento y, por lo tanto, debería mediar en la asociación entre el lazo familiar y la reincidencia. Más globalmente, el análisis persigue explorar el funcionamiento de unas políticas de reinserción que, en buena medida, atribuyen a las familias un papel protagonista en la transición postpenitenciaria.

\section{MÉTOdO}

La mayor parte de la investigación existente sobre familia y reincidencia consiste en la explotación cuantitativa de bases de datos penitenciarias que registran el número y tipo de visitas recibidas durante el cumplimiento de la pena junto con las características de las personas encarceladas y su reincidencia. Pese a la importancia de esta investigación, como ya hemos comentado en el apartado anterior estos diseños tienen limitada capacidad explicativa en tanto que no permiten identificar el mecanismo por el cual el lazo familiar incide en el proceso de desistimiento, ni el papel que tienen los aspectos subjetivos en el mismo.
Las hipótesis que se plantean en este artículo exigen un diseño que sea capaz de introducir la perspectiva del actor como elemento central del desistimiento. Para ello hemos optado por un trabajo de campo eminentemente cualitativo, mediante entrevistas biográficas, complementado con registros penitenciarios. En la fase de análisis se ha planteado una estrategia mixta; por una parte, contrastando estadísticamente hipótesis de asociación entre contactos familiares, autopercepción y reincidencia y, por otra, interpretando las narrativas de personas condenadas en su contexto biográfico. Así, el análisis cuantitativo tiene por objetivo observar si existen diferencias significativas entre dos submuestras de personas condenadas a penas de prisión según si tienen o no contacto con familiares durante el encarcelamiento; el objetivo del análisis cualitativo es explicar el porqué de estas diferencias y, por lo tanto, dotar de sentido a las asociaciones observadas cuantitativamente.

En el plano estrictamente metodológico, el artículo pretende contribuir al creciente interés en analizar muestras pequeñas mediante análisis mixtos (Small 2011), estrategia que se ha mostrado fecunda en otras investigaciones del ámbito penitenciario (ver, por ejemplo, Bahr et al. 2010; Lebel et al. 2008; Poehlmann et al. 2008). Estas aproximaciones, de carácter más intensivo que extensivo, persiguen complementar la interpretación cualitativa centrada en casos y en el punto de vista de los actores, con la comparación estadística de las diferencias existentes entre subgrupos.

\section{Campo de estudio y muestra}

Con el fin de estudiar una muestra homogénea, este proyecto se ha centrado en el perfil mayoritario entre la población encarcelada catalana y española: hombres penados por delincuencia común orientada al beneficio económico (delitos contra la propiedad y/o tráfico de drogas); se excluyen, por tanto, otros perfiles en los que los lazos familiares puede jugar un papel distinto, como es el caso de las mujeres (Almeda 2003; Giordano, Cernkovich y Rudolph 2002) y personas condenadas por otros delitos (Mears et al. 2012). Por motivos de accesibilidad y diseño de trabajo de campo, la población de referencia se restringe a la que finalizó la condena en la provincia de Barcelona entre abril y octubre de 2010.

La muestra está orientada a comparar dos grupos con distribución equivalente de edad (variable altamente asociada con la reincidencia): uno de personas clasificadas como "desistentes" y otro como "persistentes" según su autopercepción en las últimas semanas de condena (categorización realizada por los investigadores en paralelo al trabajo de campo; ver epígrafe "Variables consideradas"). Para ello los participantes se han seleccionado en dos fases: en la primera se ha pedido la participación de las personas que cumplían los criterios de inclusión y terminaban condena entre abril y mayo de 2010; en una segunda fase se seleccionaron casos específicos que finalizaban condena entre junio y octubre de 
2010; esta selección intencional tenía como objetivo obtener una muestra equilibrada en tamaño y distribución por edad de personas con autopercepción desistente y personas con autopercepción persistente. El acceso a los participantes se realizó mediante un contacto previo con ellos por parte del equipo educativo de los centros, consiguiendo, entre ambas fases, una tasa de consentimiento del $60,9 \%{ }^{3}$.

Los participantes finales fueron 67 , de los que se han eliminado 2 por información incompleta. Los 65 casos analizados tienen una distribución similar a la población estudiada según edad y grado de clasificación en el momento de finalización de la condena, con una media de 35,28 años, y encontrándose el $26,2 \%$ en libertad condicional, el $33,8 \%$ en régimen abierto (tercer grado o segundo grado semi-abierto en aplicación del art. 100.2 del Reglamento Penitenciario) y el 40,0\% en régimen cerrado (ver otros descriptivos en Tabla 1).

\section{Estrategia de integración metodológica y técnicas}

Los datos se han obtenido en dos olas. La primera ola se ha realizado semanas antes de la finalización de la condena y en ella se ha obtenido información mediante dos instrumentos: (a) entrevista biográfica semiestructurada, realizada en despachos de los centros penitenciarios o de los servicios de seguimiento de la libertad condicional, con la única presencia de entrevistado y entrevistador/a, como medio para obtener información sobre la trayectoria vital y la última etapa de condena (incluyendo los contactos con familiares), así como para identificar la autopercepción y perspectivas de los entrevistados ante la salida; (b) calendario de historia de vida (life-history calendar) (Laub y Sampson 2003; Freedman et al. 1988) para temporalizar eventos vitales a lo largo de la biografía, realizado al finalizar la entrevista biográfica; el calendario contenía cuestiones acerca de la convivencia, el lugar de residencia, la formación y el empleo, el consumo de drogas y el tiempo de encarcelamiento. La segunda ola consiste en: (c) una segunda entrevista semiestructurada, realizada entre uno y dos años después del cumplimiento de la condena, para conocer la transición prisión-libertad ( 36 de los 67 participantes han sido localizados y nuevamente entrevistados -algunos de ellos en la cárcel; otros en despachos del equipo de investigación o en sus hogares-, 2 han fallecido, 2 han rechazado la participación y el resto no han podido ser localizados); y (d) datos sobre reincidencia penitenciaria sobre todos los participantes a los 24 meses posteriores a la fecha de cumplimiento de la condena, extraídos del Servicio de Información Penitenciaria de Cataluña (SIPC) del Departamento de Justicia de Cataluña.

\footnotetext{
${ }^{3}$ No se han encontrado diferencias relevantes en el consentimiento a participar por edad o nacionalidad. En cambio, la tasa de consentimiento aumenta al $77,3 \%$ en personas que terminan la condena en régimen cerrado y disminuye al $47,7 \%$ en libertad condicional, por lo que la segunda fase de muestreo ha sobrerrepresentado esta última categoría para que tuviera un peso en la muestra equivalente al que tiene en la población de referencia.
}

Para poder contrastar estadísticamente las hipótesis planteadas se ha realizado una codificación cuantitativa de la información contenida en las entrevistas, tanto la referente a la autopercepción al final de la condena como la relativa al contacto con familiares y otros factores transicionales y biográficos. Ello permite integrar esta información con los datos cuantitativos procedentes del calendario de vida y del SIPC. Por otro lado, el análisis cualitativo de las entrevistas semiestructuradas permite identificar los mecanismos que explican las asociaciones estadísticas observadas y analizar con detalle los casos negativos.

El análisis se organiza en dos partes: La relación entre los contactos familiares durante el encarcelamiento y la autopercepción desistente (H1) es analizada estadísticamente mediante pruebas de regresión logística binaria con datos de la primera ola procedentes de las entrevistas semiestructuradas y del calendario, e interpretada mediante el análisis de contenido de las entrevistas ${ }^{4}$. El análisis de la relación entre contactos familiares, autopercepción desistente y reincidencia $(\mathrm{H} 2)$ se realiza nuevamente mediante regresión logística, tomando en consideración los datos de la primera ola y la reincidencia penitenciaria a 24 meses, mientras que la interpretación de los resultados se apoya en los materiales cualitativos de las entrevistas.

Los contrastes estadísticos se encuentran limitados por el reducido tamaño de la muestra. Por ello, se han realizado análisis exploratorios para seleccionar las variables de interés y dicotomizarlas. Asimismo, y para reducir potenciales errores de Tipo II (rechazos incorrectos de hipótesis de asociación debido al bajo tamaño muestral), se han tomado también en consideración significaciones hasta $p<0,1$.

\section{Variables consideradas en el análisis cuantitativo}

Variables dependientes: autopercepción y reincidencia Basándonos en la literatura previa sobre desistimiento, se han distinguido dos dimensiones del concepto de autopercepción: la ruptura de identidad y la autoeficacia. Por un lado, la "ruptura" con el pasado y la asunción de una identidad incompatible con la actividad delictiva (replacement self) ha sido señalada como precursor necesario del desistimiento en trabajos como los de Giordano, Cernkovich y Rudolph (2002) y Maruna (2001). Por otro, el concepto de "autoeficacia" se deriva de las contribuciones iniciales de Bandura (1977) y ha sido adaptado en investigación sobre desistimiento para referirse a la confianza en que las metas personales pueden ser logradas (Visher y O'Connell 2012; Lebel et al. 2008; Burnett y Maruna 2004; Maruna 2001).

La primera dimensión, "ruptura", se ha medido mediante una variable dicotómica que codifica si en el discurso del entrevistado hay una ruptura entre un "yo actual" que

\footnotetext{
${ }^{4}$ El análisis cualitativo de una submuestra de entrevistas de la primera ola se encuentra desarrollado en Cid y Martí (2012).
} 
aspira a asumir roles incompatibles con la delincuencia común y un "yo anterior" que ha participado en acciones delictivas. La "autoeficacia" se ha construido mediante tres códigos: la percepción de control con respecto a la capacidad para hacer efectiva una ruptura permanente con la actividad delictiva, la confianza en no volver a delinquir y la existencia de alguna estrategia -entendida como la presencia de acciones en curso o planificadas orientadas a conseguir estos objetivos (por ejemplo, búsqueda de empleo, cambio de residencia o seguimiento de programas de tratamiento de toxicomanías) ${ }^{5}$-. La variable final, "autopercepción", combina las variables "ruptura" y "autoeficacia" y permite distinguir aquellas personas con "autopercepción desistente" (esto es, entrevistados que tienen un valor positivo en la variable ruptura y, a su vez, presentan una alta autoeficacia) frente a aquellas con "autopercepción persistente".

Figura 1.

Codificación de la variable "autopercepción".

\begin{tabular}{llll}
\hline & & \multicolumn{2}{c}{ Ruptura de identidad } \\
\cline { 2 - 4 } & & Sí & No \\
\hline Autoeficacia (control, & Sí & Desistente & Persistente \\
confianza, estrategia) & No & Persistente & Persistente \\
\hline
\end{tabular}

Por otro lado, la variable "reincidencia" registra si durante los 24 meses posteriores a la finalización de la condena ha habido algún reingreso penitenciario en prisiones catalanas por nueva causa (sea como preventivo o penado). El periodo de dos años parece suficiente pues es durante el que se produce la gran mayoría de reingresos por causas nuevas: los últimos datos disponibles en Cataluña señalan que, de entre los hombres que finalizaron condena a lo largo de 2002 y habían vuelto a ingresar en prisión en un periodo de 5,5 años, el 80,4\% lo había hecho en los primeros 24 meses (Capdevila y Ferrer 2009). Con todo, la medida es limitada al no registrar delincuencia autoinformada ni encarcelamientos en prisiones de fuera de Cataluña. Con respecto a la primera cuestión, la realización de entrevistas semiestructuradas a 36 participantes en la segunda ola ha permitido considerar cualitativamente

\footnotetext{
${ }^{5}$ Estos códigos se puntuaron en una escala de tres valores, asignados mediante la interpretación y codificación del material cualitativo contenido en las entrevistas semiestructuradas. Tratándose de una codificación especialmente compleja, se ha evaluado el grado de concordancia entre dos codificadores mediante el cálculo del coeficiente de Kappa ponderado (Cohen 1968); se ha obtenido un acuerdo substancial entre codificadores, con valores de 0,88 (ruptura), 0,63 (control), 0,69 (confianza) y 0,78 (estrategia). Mediante análisis de componentes principales, las tres variables que componen la dimensión de autoeficacia $(\alpha=0,77)$ han sido reducidas a un único factor que acumula el $68,92 \%$ de la varianza inicial y que ha sido posteriormente reducido a variable dicotómica.
}

no solamente la reincidencia penitenciaria, sino también aquellas situaciones que se encuentran en un terreno intermedio entre el desistimiento y la reincidencia (reducción de la delincuencia, ponderación de la eventual comisión de delitos) e identificar los factores que están influyendo en estos procesos. Con respecto a la segunda cuestión, cabe tener en cuenta que en España, en caso que la detención y orden de ingreso penitenciario se produzca lejos del lugar de residencia, la persona suele ser trasladada a centros cercanos al mismo, y que la movilidad de los autóctonos hacia otras zonas de España es escasa; en cambio, sí es más habitual la movilidad internacional de la población inmigrante entrevistada, por lo que la reincidencia de este colectivo puede haber sido subestimada frente a la de población autóctona.

Variables independientes: contactos con familiares y otras variables consideradas

La investigación cuantitativa en el campo suele utilizar las visitas en prisión como indicador del lazo familiar durante el encarcelamiento, si bien algunos trabajos contemplan también los contactos por correo postal o telefónicos (por ejemplo, La Vigne et al. 2005). Como señalan Mills y Codd (2008) la restricción del indicador al encuentro presencial excluye otros canales que pueden ser también importantes y alternativos cuando los familiares se encuentran lejanos geográficamente. Esta cuestión es especialmente relevante en un contexto como el estudiado, caracterizado por una inmigración reciente y representando la población inmigrante una parte importante de la población encarcelada en Cataluña y también de nuestra muestra $(40,0 \%)$. A pesar de que los contactos no presenciales de las personas encarceladas con el exterior se encuentran muy restringidos, éstos son, en ocasiones, la única forma de mantener lazos con familiares ${ }^{6}$.

Por ello hemos adoptado una aproximación transnacional al contacto con familiares adultos en la que, a partir de la información de las entrevistas, registramos si existe contacto regular con los "padres" (padre y/o madre), "pareja" (relación estable de un año o más de duración) u "otros familiares adultos" (hermanos/as, primos/as, tíos/as, parientes políticos, entre otros), sea de forma presencial o telefónica, durante la última etapa de la condena en régimen ordinario (e independientemente de que estos familiares

\footnotetext{
${ }^{6}$ Con todo, y a diferencia de estudios realizados en años anteriores (p. ej., García-Borés et al. 2006), en nuestra muestra no es habitual que la población inmigrante carezca de lazos familiares en Cataluña. Típicamente, una parte de la red familiar forma parte de la cadena migratoria, ha emigrado a Cataluña antes, simultáneamente o después que el entrevistado y mantiene contacto presencial con él, mientras que otra se encuentra en el país de origen y/o en otros países europeos o en EE. UU. Estos lazos físicamente distantes pueden, aun sin contacto presencial, prestar apoyo instrumental y expresivo, ser fuente de vínculo y/o de cambio cognitivo.
} 
se encuentren en el lugar de residencia, en el de origen 0 en otros destinos migratorios).

La medición del contacto con menores es más problemática. Por lo que se refiere al contacto presencial, García-Borés et al. (2006) señalan la deficitaria adecuación del sistema de visitas a menores de edad, y el frecuente rechazo familiar al contacto de menores con la cárcel. Además, parece complicado delimitar una medida estandarizada de contacto que sea a la vez válida para bebés, niños, adolescentes y mayores de edad, pues en unos casos se depende de familiares adultos para mantener este contacto y en otros no. Por ello, se ha optado por considerar no únicamente el contacto directo sino también el indirecto a través de referentes adultos.

Finalmente, es importante mencionar que aunque en el análisis nos centramos en términos operativos en la presencia de contacto, entendemos este como un indicador del lazo familiar, que es obviamente más amplio que el mero contacto.

Adicionalmente se han tenido en cuenta otras variables a efectos de exploración y control:

(a) Recursos económicos al finalizar la condena. Medidos mediante dos variables: "ingresos regulares" se refiere a si el entrevistado percibe regularmente rentas derivadas del empleo o algún tipo de subsidio permanente (por ejemplo, incapacidad permanente o jubilación) ${ }^{7}$; "vivienda" registra si la persona tiene acceso a una vivienda en Cataluña al finalizar la condena, propia o familiar.

(b) Consumo de drogas, considerando dos variables dicotómicas: el "consumo anterior" (si la persona ha consumido heroína o cocaína regularmente con anterioridad -durante dos o más años-) y si es "consumidora actual".

(c) Delincuencia y prisión: edad del "primer delito", edad del "primer ingreso en prisión", existencia de "ingresos penitenciarios anteriores" y "tiempo relativo en prisión"8.

(d) Otras variables: "país de nacimiento" (España, extranjero); "años de educación reglada"; "años de experiencia

\footnotetext{
${ }^{7}$ Se han agregado en una misma variable los datos de empleo y pensiones debido a que son pocos los entrevistados que cuentan con empleo al finalizar la condena. Por otro lado, no se ha considerado el subsidio de excarcelación por ser una prestación temporal.

${ }^{8}$ Esta variable se ha obtenido a partir de lo declarado por los entrevistados en el calendario de historia de vida, que registra el número de años en los que se ha estado, en algún momento, cumpliendo condena de prisión en primer, segundo o tercer grado. Se ha descartado la utilización de los datos del historial penitenciario procedentes del SIPC porque no contemplan los encarcelamientos en prisiones no catalanas. Por otro lado, y puesto que la magnitud de esta última variable está afectada por la edad (los de mayor edad tienen una mayor probabilidad de haber pasado más tiempo encarcelados), se ha considerado el tiempo relativo en prisión sobre la edad adulta.
}

laboral" (considerando el número de años en los que ha estado desarrollando un trabajo remunerado de 10 o más horas semanales durante al menos 6 meses a lo largo del año); y existencia de "antecedentes de delincuencia familiar" (antecedentes delictivos contra la propiedad o tráfico de drogas en la familia nuclear de origen).

Por lo que respecta a la "edad", el diseño muestral ha forzado que ambas submuestras tengan una distribución similar, con lo que la variable no muestra diferencias significativas entre los grupos (ver tabla 1).

\section{Resultados}

\section{Contactos familiares y autopercepción desistente}

En un primer nivel de análisis se exploran las asociaciones bivariadas de las variables independientes con la autopercepción. Como se observa en la Tabla 1, los contactos con familiares, considerados globalmente, están asociados a la autopercepción desistente (y esta con la reincidencia penitenciaria). Particularmente, son los contactos con los padres y con la pareja los que presentan una asociación significativa, mientras que la asociación con otros familiares es más débil y la relación con "contacto con hijos" no es estadísticamente relevante.

Por lo que se refiere a los recursos económicos con los que se cuenta a la salida, el hecho de disponer de fuentes de renta (derivadas del empleo o de subsidios por incapacidad laboral o jubilación) es un factor altamente asociado a la autopercepción desistente; mientras que el tener acceso a una vivienda no discrimina entre ambos grupos, como tampoco el consumo de drogas actual o anterior (que, pese a mostrar una mayor intensidad en la asociación, no llega a ser significativa).

Por otro lado, y como es esperable, el tiempo de encarcelamiento y la experiencia laboral aparecen altamente relacionadas con la autopercepción: son factores que configuran la identidad y que afectan claramente a la percepción de autoeficacia.

La exploración bivariada ha servido no solamente para tener una primera idea de las asociaciones de las variables consideradas con la autopercepción, sino también para seleccionar aquellas que se van a introducir en el modelo multivariado -necesariamente limitado debido al reducido tamaño de la muestra ${ }^{9}$ - y comprobar si la asociación entre

\footnotetext{
${ }^{9}$ Con respecto al número máximo de variables a introducir en los modelos de regresión logística, Vittinghoff y McCulloch (2007) muestran que el criterio convencional de contemplar un mínimo de 10 eventos por variable independiente es excesivamente conservador, y que los resultados se mantienen estables con 5 eventos por variable. Con todo, y para minimizar los posibles sesgos derivados del bajo número de eventos en la muestra analizada, los modelos contemplan
} 
Tabla 1.

Descriptivos y asociaciones bivariadas con la autopercepción desistente (Phi).

\begin{tabular}{|c|c|c|}
\hline & $\mathrm{n}(\%)$ & Phi \\
\hline Autopercepción desistente & $34(52 \%)$ & - \\
\hline Reincidencia penitenciaria & $15(24 \%)$ & $-0,471^{* * *}$ \\
\hline \multicolumn{3}{|l|}{ Contacto con familiares } \\
\hline Padres & $35(54 \%)$ & $0,290^{* *}$ \\
\hline Pareja & $24(37 \%)$ & $0,284^{* *}$ \\
\hline Hijos/as & $11(17 \%)$ & 0,185 \\
\hline Otros familiares & $24(37 \%)$ & $0,220^{*}$ \\
\hline Cualquier familiar & $48(74 \%)$ & $0,343^{* * *}$ \\
\hline \multicolumn{3}{|l|}{ Recursos económicos a la salida } \\
\hline Ingresos regulares & $19(29 \%)$ & $0,343^{* * *}$ \\
\hline Vivienda & $53(82 \%)$ & $-0,057$ \\
\hline \multicolumn{3}{|l|}{ Drogas } \\
\hline Consumo anterior & $38(59 \%)$ & $-0,180$ \\
\hline Consumidor actual & $11(17 \%)$ & $-0,144$ \\
\hline \multicolumn{3}{|l|}{ Delincuencia } \\
\hline Primer delito con 18 años o más años & $27(42 \%)$ & $0,305^{\star *}$ \\
\hline Primer ingreso en prisión con 22 años o más & $41(63 \%)$ & $0,291^{* *}$ \\
\hline Ingresos penitenciarios anteriores & $34(52 \%)$ & $-0,233^{*}$ \\
\hline Tiempo relativo en prisión superior a la media & $29(45 \%)$ & $-0,444^{* * *}$ \\
\hline \multicolumn{3}{|l|}{ Otras variables } \\
\hline País de nacimiento (España) & $39(60 \%)$ & $-0,151$ \\
\hline Años de educación reglada (más de 8) & $36(55 \%)$ & 0,196 \\
\hline Años de experiencia laboral (superior a la media) & $22(34 \%)$ & $0,488^{* * *}$ \\
\hline Antecedentes delincuencia familiar & $17(26 \%)$ & $-0,063$ \\
\hline Edad al finalizar condena (media, desv.) & $35,28(10,0)$ & Eta $=.141$ \\
\hline
\end{tabular}

$n=65,{ }^{*} p<0,1 ;{ }^{* *} p<0,05 ;{ }^{* * *} p<0,01$ en prueba de Chi-Cuadrado.

contactos familiares y autopercepción se mantiene bajo el control de otras variables. Por ello, nos centraremos específicamente en el control por "ingresos regulares" como indicador de recursos económicos a la salida y en el "tiempo relativo en prisión" como indicador de trayectoria ${ }^{10}$. Por lo que refiere a los contactos familiares, nos centraremos en los contactos con "padres", "pareja" y "otros familiares",

la inclusión por separado de cada una de las tres variables consideradas de contactos familiares. En el caso de los contrastes de reincidencia, en los que el número de eventos es particularmente bajo, adicionalmente al control por dos variables se han replicado los modelos con una sola variable de control (modelos no incluidos en el artículo) confirmando que los efectos analizados se mantenían estables.

10 Pese a la alta significación de la experiencia laboral, se ha descartado su inclusión en los modelos por estar relacionada con el tiempo de encarcelamiento (siendo esta última variable la habitualmente utilizada en investigación sobre reincidencia). Con todo, si en los modelos se substituye el tiempo de encarcelamiento por la experiencia laboral no se observa ningún cambio substancial en los resultados. Igualmente, se han explorado modelos con las otras variables de control consideradas en la Tabla 1, obteniéndose efectos similares de los contactos familiares. En ninguno de los modelos se han detectado problemas de multicolinealidad. descartando la variable "contacto con hijos" en tanto que se ha mantenido no significativa en todos los modelos ejecutados.

La tabla 2 presenta cuatro modelos de regresión logística, los tres primeros introduciendo un tipo de contacto familiar junto con las otras variables consideradas, y el cuarto integrando los tres tipos de contacto en el modelo. Se confirma la asociación positiva entre los contactos regulares con padres y parejas y la autopercepción desistente al finalizar la condena (no los contactos con otros familiares), una vez controlados los efectos de ingresos y tiempo de encarcelamiento.

El análisis cualitativo de las entrevistas permite identificar dos mecanismos que vinculan los contactos familiares con la autopercepción (véase Cid y Martí 2012 para un mayor desarrollo). El primero, que se da entre los entrevistados más jóvenes, correspondería a lo que Sampson y Laub (1993) denominan puntos de inflexión: una nueva relación de pareja que desaprueba la actividad delictiva y que apoya a la persona durante el encarcelamiento; es el compromiso con esta relación el que motiva al entrevistado al desistimiento: 
Tabla 2.

Regresión logística sobre autopercepción desistente.

\begin{tabular}{|c|c|c|c|c|c|c|c|c|}
\hline & \multicolumn{2}{|c|}{ Modelo 1} & \multicolumn{2}{|c|}{ Modelo 2} & \multicolumn{2}{|c|}{ Modelo 3} & \multicolumn{2}{|c|}{ Modelo 4} \\
\hline & Coef. & E & Coef. & E & Coef. & $E$ & Coef. & E \\
\hline \multicolumn{9}{|l|}{ Contacto familiar } \\
\hline Padres & $1,830^{\star \star *}$ & 0,686 & & & & & $1,563^{* *}$ & 0,719 \\
\hline Pareja & & & $1,626^{* *}$ & 0,677 & & & $1,750^{* *}$ & 0,784 \\
\hline Otros familiares & & & & & 0,871 & 0,609 & 0,950 & 0,713 \\
\hline \multicolumn{9}{|l|}{ Variables control } \\
\hline Ingresos regulares & $1,674^{* *}$ & 0,745 & $1,967^{\star *}$ & 0,761 & $1,409^{* *}$ & 0,687 & $2,163^{* * *}$ & 0,826 \\
\hline Tiempo en prisión & $-2,132^{* * *}$ & 0,673 & $-1,664^{* * *}$ & 0,612 & $-1,750^{* * *}$ & 0,593 & $-1,945^{\star \star *}$ & 0,704 \\
\hline Constante & $-0,377$ & 0,544 & $-0,260$ & 0,530 & 0,176 & 0,478 & $-1,375^{*}$ & 0,742 \\
\hline Chi-cuadrado & $26,656^{* * *}$ & & $24,534^{* * *}$ & & $20,310^{* * *}$ & & $33,018^{* * *}$ & \\
\hline$R^{2}$ de Nagelkerke & 0,449 & & 0,419 & & 0,358 & & 0,531 & \\
\hline
\end{tabular}

${ }^{*} p<0,1 ;{ }^{* *} p<0,05 ;{ }^{* * *} p<0,01$.

"Por qué cambié? Porque conocí a la chica esta con la que estoy, me metió en la cabeza que no tenía que hacer estas cosas, que...." (E28).

El segundo corresponde a lazos con parejas o padres que ya existían cuando tuvieron lugar las acciones por las que los entrevistados han sido condenados, pero que en su momento no fueron factor de prevención ni ejercieron de punto de inflexión; en estos casos el efecto del contacto durante el encarcelamiento se observa de forma especialmente clara, porque los entrevistados plantean el deber de compensar a los familiares -especialmente mujeres, que son quienes protagonizan primordialmente el contactopor su apoyo durante esta etapa, "demostrándoles" que pueden dar un giro a su trayectoria una vez en libertad:

\footnotetext{
"... lo hago por ellos [mis padres], momentos de no puedo más, no quiero que tampoco... el delito lo he hecho yo, no lo han hecho ellos, no hay derecho tampoco a que tengan que estar todos los fines de semana en una cárcel ellos, ellos no... no se lo merecen". (E338).
}

El apoyo emocional que implica el contacto en sí mismo suele ir acompañado de un apoyo instrumental (ingresos de peculio, vivienda y manutención durante los permisos o estando en régimen abierto) con expectativas de continuidad en el futuro. Pero incluso sin este apoyo instrumental y sin contacto presencial, como es el caso de las personas cuyos padres están en el país de origen, el mantenimiento del lazo acaba provocando el deseo de compensación:

"Cuando cobro intento llamarles, para que no se preocupen de mí. Y ellos saben que me queda poco, y me están diciendo 'llévatelo bien' .... Y yo intento llevarlo lo mejor posible, porque me queda poco y, no quiero causar más daño a mi madre, porque... ya hay suficiente. Con dos hijos en la cárcel, uno en la calle, y mi padre se fue de casa cuando yo tenía ocho años, no, no me acuerdo, a lo mejor con cinco años. Y la pobre está, está...." (E314).
En definitiva, los resultados obtenidos confirman y perfilan la primera hipótesis planteada sobre asociación entre contactos familiares durante el encarcelamiento y autopercepción desistente a la salida. Particularmente, son los contactos con padres y parejas los que tienen un efecto relevante sobre la autopercepción desistente. Por su parte, el análisis cualitativo muestra dos mecanismos por los que se produce esta asociación: compromiso y compensación.

Asimismo, las entrevistas permiten observar con mayor detalle los casos negativos o no confirmatorios e identificar otros factores influyentes. Por un lado están aquellas personas que han construido una autopercepción desistente sin tener contactos familiares activos; por otro los que, a pesar de contar con estos lazos, tienen una autopercepción persistente.

Con respecto a los primeros (personas con autopercepción desistente aun sin contactos familiares activos) pueden identificarse básicamente dos situaciones. La primera corresponde a entrevistados con alta experiencia laboral (esta es una situación habitual en inmigrantes que tienen un inicio tardío en la delincuencia motivado primordialmente por necesidad económica en la postmigración y que también se produce en casos de delincuencia ocasional): en estos perfiles prima una identidad trabajadora y una elevada percepción de autoeficacia derivada de la experiencia laboral, de forma que la autopercepción no está necesariamente asociada al vínculo familiar:

"... no vas a estar pendiente de tu familia, yo nunca estuve pendiente de mi familia, salvo a los, los 4 primeros meses, el resto para mi, todo lo... lo generaba yo". (E143).

La segunda corresponde a personas a partir de 50 años que plantean una reflexión sobre su ciclo vital y en 
las que un eventual retorno a prisión se percibe como algo todavía más costoso que entre las personas jóvenes:

"Tengo 50 años y no se qué es vivir la vida. Entonces, si quiero vivir un poco la vida tengo que espabilarme". (E164).

Con respecto a las personas que, pese a tener contactos familiares activos, no han desarrollado una autopercepción desistente, el análisis cualitativo muestra que estos lazos, a diferencia de los anteriores, no son percibidos por los entrevistados en términos de exigencia al cambio (esto es, de compromiso o compensación hacia quien presta el apoyo):

"... cuando salí igual, [mi hermano] me lo volvió a repetir: 'Tienes que cambiar de vida, no puedes seguir, no puedes volver a entrar en prisión. Corres el tiempo de que si vuelves a entrar en prisión y te meten una condena muy grande no vuelvas a salir de ella. Entonces... mira pos por ti mismo, no es por nadie más. Simplemente por ti, porque nosotros seguiremos con nuestras vidas, ¿me entiendes?'. Pero uno sí, uno escucha y eso, pero quizá no escucha lo suficiente. Y bueno, es, es lo que me ha tocado vivir, en principio". (E53).

Aunque estas situaciones pueden reflejar un debilitamiento del lazo familiar que se ha producido desde la adolescencia con el inicio de la trayectoria delictiva, las entrevistas muestran como los largos tiempos de encarcelamiento contribuyen a este debilitamiento, aun manteniendo el contacto:

\footnotetext{
"... las visitas están limitadas, son tan cortas, tan... que se ha perdido ese vínculo de familia, familia ¿Vale? O sea, no es lo mismo estar 20 años cada día levantándote y acostándote con tu familia a estar 20 años pues viniéndote a ver una vez a la semana cuando pueden. ¡Y veinte minutos! Por un cristal... ¡No es lo mis$\mathrm{mo}$ ! El vínculo se va perdiendo. El roce hace el cariño al final y eso se pierde". (E53).
}

En otros casos, especialmente en los entrevistados más jóvenes, el vínculo con padres o parejas no es lo suficientemente fuerte para ejercer de punto de inflexión frente a la influencia de una red amical criminógena:

\begin{abstract}
“... si encuentro trabajo pues... ya estaría bien, me entiendes, me plantaría aquí, con mi familia y...... y acabar... acabar con todo, me entiendes, esto... el tiempo que he estado aquí......, ya la diversión prácticamente se me habría terminado, pero eso... eso nunca lo sabe uno hasta que no llega a la calle [sonríe], me entiendes, eso es un problema también". (E5).
\end{abstract}

\section{Reincidencia, autopercepción desistente y contactos familiares}

En este subapartado se analiza si la autopercepción media la relación entre contactos familiares y reincidencia penitenciaria a los 24 meses de la finalización de la condena. Los casos válidos para este análisis se reducen a 62 , puesto que 2 de los 65 entrevistados han fallecido durante estos 24 meses y 1 todavía no había sido excarcelado al haber sido condenado por otras causas antiguas justo antes de finalizar la anterior condena. De las 62 personas restantes, hay $15(24 \%)$ que han reingresado en prisiones catalanas por causas nuevas (en situación de presos preventivos o condenados).

La hipótesis de la que partimos es que los contactos con los padres y las parejas durante el encarcelamiento son explicativos de la no-reincidencia en el periodo de seguimiento. Además, en la medida en que consideramos que la autopercepción desistente conforma el vínculo explicativo, entonces la autopercepción debería mediar la relación entre ambos factores (contactos familiares y noreincidencia).

Los modelos de la tabla 3 confirman la asociación entre autopercepción y reincidencia, mientras que el resto

Tabla 3.

Regresión logística sobre reincidencia penitenciaria a 24 meses (con autopercepción).

\begin{tabular}{|c|c|c|c|c|c|c|c|c|}
\hline & \multicolumn{2}{|c|}{ Modelo 1} & \multicolumn{2}{|c|}{ Modelo 2} & \multicolumn{2}{|c|}{ Modelo 3} & \multicolumn{2}{|c|}{ Modelo 4} \\
\hline & Coef. & $\mathrm{E}$ & Coef. & E & Coef. & E & Coef. & E \\
\hline Autopercepción & $-2,136^{* *}$ & 0,942 & $-2,225^{\star *}$ & 0,958 & $-1,897^{\star *}$ & 0,904 & $-2,199^{\star *}$ & 1,033 \\
\hline \multicolumn{9}{|l|}{ Contacto familiar } \\
\hline Padres & 0,281 & 0,754 & & & & & 0,485 & 0,809 \\
\hline Pareja & & & 0,531 & 0,830 & & & 0,244 & 0,856 \\
\hline Otros familiares & & & & & $-1,247$ & 0,897 & $-1,298$ & 0,944 \\
\hline \multicolumn{9}{|l|}{ Variables control } \\
\hline Ingresos regulares & $-1,302$ & 1,169 & $-1,164$ & 1,187 & $-1,307$ & 1,191 & $-1,261$ & 1,219 \\
\hline Tiempo en prisión & 0,847 & 0,770 & 0,898 & 0,756 & 0,746 & 0,783 & 0,616 & 0,824 \\
\hline Constante & $-0,707$ & 0,683 & $-0,768$ & 0,696 & $-0,262$ & 0,732 & $-0,393$ & 0,788 \\
\hline Chi-cuadrado & $17,932^{\star \star *}$ & & $18,211^{\star \star *}$ & & $19,961^{* \star *}$ & & $20,454^{\star * *}$ & \\
\hline $\mathrm{R}^{2}$ de Nagelkerke & 0,375 & & 0,380 & & 0,411 & & 0,420 & \\
\hline
\end{tabular}

${ }^{*} p<0,1 ;{ }^{* *} p<0,05 ;{ }^{* * *} p<0,01$. 
de variables -incluidos los contactos familiares- no tiene ningún efecto significativo. Esto es: para una parte importante de los entrevistados, la reincidencia a los 24 meses se explica por la autopercepción que tenían al finalizar el encarcelamiento (en cuya construcción han tenido un papel relevante, junto a otros factores, los contactos familiares). Asimismo, es de interés observar los coeficientes correspondientes a los distintos contactos familiares. En primer lugar, los contactos con padres y pareja, aun sin ser significativos, están "positivamente" relacionados con la reincidencia. Esto nos indica que estos lazos pueden tener efectos de signo contrario: por un lado, el contacto durante el encarcelamiento fortalece el vínculo y apoya el cambio cognitivo (y, por lo tanto, interviene indirectamente reduciendo la posibilidad de reincidencia); por otro lado, favorecen la reincidencia (de forma directa y una vez controlados los efectos de la autopercepción). En segundo lugar, los contactos con otros familiares, aunque tampoco significativos, son los que tienen un mayor peso directo en reducir la probabilidad reincidencia. Discutimos más adelante estos resultados.

La Tabla 4 reproduce los mismos modelos que la Tabla 3 excluyendo la variable autopercepción. Contrariamente a lo planteado en la segunda hipótesis, se observa, en primer lugar, que los contactos con padres y pareja no tienen ninguna asociación apreciable sobre la reincidencia, lo que se explicaría por el doble efecto (negativo e indirecto -mediado por la autopercepción- y positivo y directo) identificado en la Tabla 3. En segundo lugar, se observa nuevamente que el coeficiente más alto -y significativo al $90 \%$ - corresponde a los contactos con otros familiares. Ello plantea dos cuestiones: (1) ¿por qué el contacto con padres o parejas, que estaba altamente asociado a la autopercepción desistente, no lo está de forma directa con la no-reincidencia?, y (2) ¿por qué el contacto con otros familiares, que no era relevante para

explicar la autopercepción desistente, pasa a estar significativamente asociado a la no-reincidencia?

Con respecto a la primera cuestión (ausencia de relación directa entre lazos con padres/parejas y reincidencia), las entrevistas realizadas en la segunda ola muestran nuevamente patrones de desistimiento que no pasan por el apoyo familiar. No se trata solamente de las personas con mayor experiencia e identidad laboral, sino también de adultos con más tiempo de encarcelamiento que, aun encontrándose en situación de exclusión al finalizar la condena, descartan asumir cualquier riesgo que les pueda conducir nuevamente a la cárcel, como la vuelta al consumo de drogas:

\footnotetext{
"... es que no quiero liarme, ¿sabes?, porque nadie lía a nadie, pos si lo ves, y te pilla con dinero, 'Oye, hay un hueco', pos venga, y hueco casi siempre hay, entonces pues... ¿sabes? Ya intento no ver a los que tienen coche, pa', para no liarme, sobre todo cuando tengo dinero. Pago la habitación y me gasto el dinero lo antes posible, compro tabaco para el mes, comida y eso, y me quedo sin dinero lo antes posible para no tener dinero y no consumir, ¿sabes? Porque no sé si tuviera dinero cada día, no sé si consumiría o no, ¿sabes? Y... estoy así, estoy indeciso, estoy que en la cabeza no sé para dónde tiro, no sé lo que hacer, no sé... A ver, si no quiero volver alli a... a la cárcel no quiero volver, porque no estoy para las cárceles ya, no tengo la fuerza necesaria para estar allí". (E330).
}

En segundo lugar, el análisis cualitativo muestra que los lazos familiares pueden ser un factor de tensión, concretamente cuando su apoyo instrumental en la etapa postpenitenciaria no es suficiente para cubrir las necesidades básicas. De hecho, incluso puede implicar una mayor exigencia económica para el excarcelado si este se percibe como el responsable de la manutención de sus familiares, sea en el país de origen (mediante remesas) 0 en Cataluña. Esta tensión económica se observa especialmente en el caso de roles paternos:

Tabla 4.

Regresión logística sobre reincidencia penitenciaria a 24 meses (sin autopercepción).

\begin{tabular}{|c|c|c|c|c|c|c|c|c|}
\hline & \multicolumn{2}{|c|}{ Modelo 1} & \multicolumn{2}{|c|}{ Modelo 2} & \multicolumn{2}{|c|}{ Modelo 3} & \multicolumn{2}{|c|}{ Modelo 4} \\
\hline & Coef. & E & Coef. & E & Coef. & E & Coef. & $E$ \\
\hline \multicolumn{9}{|l|}{ Contacto familiar } \\
\hline Padres & $-0,316$ & 0,654 & & & & & $-0,093$ & 0,691 \\
\hline Pareja & & & $-0,128$ & 0,702 & & & $-0,233$ & 0,732 \\
\hline Otros familiares & & & & & $-1,456^{*}$ & 0,855 & $-1,461^{*}$ & 0,871 \\
\hline \multicolumn{9}{|l|}{ Variables control } \\
\hline Ingresos regulares & $-1,845^{*}$ & 1,103 & $-1,887^{*}$ & 1,115 & $-1,711$ & 1,117 & $-1,760$ & 1,131 \\
\hline Tiempo en prisión & $1,512^{* *}$ & 0,681 & $1,491^{* *}$ & 0,691 & $1,379^{\star}$ & 0,703 & $1,341^{*}$ & 0,714 \\
\hline Constante & $-1,465^{* *}$ & 0,642 & $-1,563^{\star *}$ & 0,654 & $-1,172^{\star}$ & 0,603 & $-1,013$ & 0,740 \\
\hline Chi-Cuadrado & $11,559^{\star \star \star *}$ & & $11,358^{\star *}$ & & $14,774^{\star \star *}$ & & $14,909^{\star *}$ & \\
\hline $\mathrm{R}^{2}$ de Nagelkerke & 0,254 & & 0,250 & & 0,317 & & 0,319 & \\
\hline
\end{tabular}

${ }^{*} p<0,1 ;{ }^{* *} p<0,05 ;{ }^{* * *} p<0,01$. 
"[Mi mujer] claro, se enfadó, se enfadó: 'Que no, que puedes volver a la cárcel otra vez, podemos comer pan y agua', pero pan y agua para ti y para mí, pero para los críos... necesitan leche, necesitan cereales, sus cosas, su almuerzo para el cole, necesitan esto y lo otro; ella nunca ha estado de acuerdo, pero bueno, y nunca me ha cogido ese dinero, por ejemplo lo dejo en el cajón y voy comprando del súper y tal, pero digo 'Toma', nunca quiere el dinero, porque no le gustaba...." (E360).

Para visualizar el efecto específico de la paternidad hemos ejecutado nuevamente el Modelo 2 de la Tabla 4 añadiendo la variable "contacto con hijos" (no mostrado en la tabla): el coeficiente de regresión correspondiente a la variable "contacto con pareja" pasa de $-0,128$ a $-0,568$, mientras que el de "contacto con hijos" es de $+0,953$. Aunque ninguno de ellos es significativo, el signo de los coeficientes sugiere que serían estos últimos quienes podrían implicar un aumento de la probabilidad de reincidencia, mientras que la relación de pareja mantendría cierto papel "protector".

En todo caso, es relevante señalar como la exigencia de generar ingresos está principalmente asociada a la figura del proveedor masculino, de forma que la presión en esta cuestión se dirige hacia el ex-preso:

"[Mi compañera] no lo quiere que lo haga [robar], pero ella también tiene que pensar que yo me considero una persona responsable, y que no me gusta que me mantengan, entonces yo no puedo estar en mi casa así parado, que ella está trabajando y está trayendo dinero a la casa, y no me gusta, ¿sabes? Aunque yo trabajara, trabajaríamos los dos, y vayamos la casa p'alante y todo p'alante entre los dos, pero yo sí. A lo mejor puedo estar un tiempo sin hacer nada, pero cuando tenemos una discusión, siempre sale el tema, entonces ya... pues ya me quemo, porque tiene razón, que a lo mejor a veces también me dice que no lo haga pero luego me obliga, ¿no?, me dice 'Que no tienes trabajo', pum, entonces yo me siento obligado, ¿sabes?”. (E211).

Con respecto a la segunda cuestión planteada (relevancia de los contactos con otros familiares para explicar la no-reincidencia) nos hemos fijado en la transición de aquellas personas que tuvieron contacto con otros familiares y no han reincidido (de los 21 casos que se encuentran en esta situación, 9 han sido entrevistados en la segunda ola) para identificar el papel que han jugado estos lazos. Las entrevistas muestran que mayoritariamente han sido fuente de apoyo, ofreciendo en algunos casos alojamiento, en otros transferencias dinerarias puntuales para cubrir necesidades básicas e incluso inserción laboral. En varios casos son hermanas (más que hermanos) quienes prestan apoyo. Pero este se extiende, con independencia del género, a familiares más lejanos con los que no necesariamente se mantuvo contacto durante el encarcelamiento, pero cuya ayuda evita situaciones críticas:

"... un cuñado mío ... hace poco tuvo que prestarnos, o regalarnos, 1000 euros, porque estábamos hasta las... con deudas y eso, nos mandó 1000 euros... un familiar nuestro ... Sí, es muy, muy doloroso, pero bueno. No hay otra alternativa, ¿qué, qué hacemos?" (E206).

Ello sugeriría que el papel de estos familiares no consiste tanto en catalizar un cambio cognitivo durante el encarcelamiento, sino en actuar como apoyo en la etapa postpenitenciaria.

\section{CONCLUSIONES}

En este artículo nos hemos preguntado por el efecto que tienen los lazos familiares durante el encarcelamiento en la autopercepción desistente y en la reincidencia de una muestra de hombres condenados a prisión en la provincia de Barcelona por delincuencia común orientada al beneficio económico. El análisis pretende ir más allá de contrastar la existencia de asociación entre familia y reincidencia, para abordar de qué forma estos lazos inciden en el proceso de transición a la vida en libertad, en un contexto en el que las familias ejercen un rol central en la provisión de bienestar. Para ello se ha planteado un diseño mixto en el que se han obtenido datos cualitativos y cuantitativos para un mismo conjunto de casos. Este diseño muestra su capacidad explicativa para entender la relación entre factores objetivos y subjetivos en el proceso de desistimiento y situarlos en el contexto de trayectorias particulares.

Los resultados confirman la primera hipótesis relativa a que mantener el contacto con pareja y/o padres durante el encarcelamiento está altamente asociado a una autopercepción desistente al finalizar la condena. Particularmente, y más allá del apoyo que estos lazos pueden ofrecer (en el sentido de Wright, Cullen y Miller 2001), el contacto durante el encarcelamiento crea también un vínculo (en el sentido de Sampson y Laub 1993) al provocar el deseo de compensar a los familiares por este apoyo o bien de comprometerse con una relación de pareja. Y este deseo contribuye a generar una autopercepción desistente. Asimismo, el análisis permite introducir varios matices a esta afirmación: en primer lugar, que la capacidad que tienen los lazos familiares para construir o fortalecer un vínculo es menor en ciertas condiciones, como el largo tiempo de encarcelamiento o la influencia de un entorno amical criminógeno (en los más jóvenes); en segundo lugar, que el contacto con familiares no es la única vía hacia el desistimiento: la experiencia laboral (mayor en aquellos que han estado menos tiempo encarcelados) dota a los entrevistados de identidad trabajadora y mayor percepción de autoeficacia, lo que facilita la ruptura con la etapa penitenciaria; por otro lado, la reflexión sobre el ciclo vital iniciada en la madurez impulsa al cambio independientemente de los apoyos familiares -aspecto ya señalado por Shover (1985)-.

Con respecto a la segunda hipótesis (sobre si la autopercepción desistente media en la relación entre contactos familiares y reincidencia), los resultados muestran que la 
autopercepción es un buen predictor de la futura no-reincidencia. Sin embargo, y contrariamente a lo planteado, los lazos con parejas y padres no están asociados a la reincidencia: si detrás de ellos no hay un apoyo instrumental, la transición puede truncarse. Además, la concepción de la figura masculina como principal proveedora dineraria del hogar, muy presente en los entrevistados, sitúa sobre los hombres excarcelados la presión de generar ingresos, siendo un colectivo con enormes dificultades de inserción laboral. Los lazos con otros familiares, en cambio, pueden ser fuente de apoyo material y expresivo durante el postencarcelamiento sin las exigencias que puede comportar el vínculo con familares más directos, como sugieren los trabajos de Duwe y Clark (2013) y Berg y Huebner (2011).

En síntesis, mientras que los resultados de la primera hipótesis son un reflejo del familismo y su "marca" de género -son las mujeres (no solo como parejas, también como madres) quienes protagonizan el apoyo y la construcción de vínculo-, los de la segunda muestran sus límites: el vínculo familiar, aunque importante, no es suficiente si no puede aportar el apoyo instrumental necesario para acompañar la transición postpenitenciaria.

Si el vínculo familiar es relevante, parece pertinente promover políticas de reinserción que faciliten el contacto cotidiano de la persona con sus redes más próximas. No obstante, dejar las políticas de reinserción a merced de las familias no solo carga sobre ellas el peso de la excarcelación, sino que también tiene importantes limitaciones: por una parte existirán personas que carezcan de estos vínculos; por otra existirán casos en que el vínculo familiar no sea suficiente para generar procesos de desistimiento; y finalmente la familia puede carecer de los recursos suficientes para prestar el apoyo necesario y ser por tanto incapaz de contribuir al desistimiento. Cabe, por último, considerar el contexto socio-económico con el que se encuentran los entrevistados en esta investigación al salir de la cárcel: alto desempleo, desalojos de vivienda, recortes en políticas sociales; esto es, un contexto con un bajo nivel de apoyo social formal (Cullen 1994) en el que, si no se dispone de apoyos informales, es muy complicado materializar un proceso de desistimiento sin caer en exclusión.

\section{RefERENCIAS BibLIOGRÁficAs}

Almeda, E. 2003. Mujeres encarceladas. Barcelona: Ariel.

Alós, R., F. Esteban, P. Jódar, F. Miguélez, V. Alcaide i P. López-Roldán. 2011. La inserció laboral dels exinterns dels centres penitenciaris de Catalunya. Barcelona: Centre d'Estudis Jurídics i Formació Especialitzada.

Bahr, S., L. Harris, J. K. Fisher and A. H. Armstrong. 2010. "Successful reentry: what differentiates successful and unsuccessful parolees?". International Journal of Offender Therapy and Comparative Criminology 54:667-692. http://dx.doi.org/10.1177/0306624X09342435
Bales, W. and D. Mears. 2008. "Inmate social ties and the transition to society: does visitation reduce recidivism?". Journal of Research in Crime and Delinquency 45:287-321. http://dx.doi.org/10.1177/0022427808317574

Bandura, A. 1977. "Self-efficacy: Towards a unifying theory of behavioural change". Psychological Review 84:191-215. http://dx.doi.org/10.1037/0033-295X.84.2.191

Berg, M. and B. Huebner. 2011. "Reentry and the ties that bind: An examination of social ties, employment, and recidivism". Justice quarterly 28:382-410. http://dx.doi.org/10.1080/07418825.2010.498383

Burnett, R. 1992. The dynamics of recidivism. Oxford: Centre for Criminological Research.

Burnett, R. and S. Maruna. 2004. "So 'prison works', does it? The criminal careers of 130 men released from prison under Home Secretary Michael Howard". The Howard Journal of Criminal Justice 43:390-404. http://dx.doi.org/10.1111/j.1468-2311.2004.00337.x

Calverley, A. 2013. Cultures of desistance: rehabilitation, reintegration, and ethnic minorities. London: Routledge.

Capdevila, M. y M. Ferrer. 2009. "Tasa de reincidencia penitenciaria 2008". Documento de trabajo (Investigación propia, 2008), Centre d'Estudis Jurídics i Formació Especialitzada, Barcelona. Consulta 24 de diciembre de 2012 http://www20.gencat.cat/ docs/Justicia/Documents/ARXIUS/SC-1-076-09_cas.pdf).

Castillo, J. y M. Ruiz. 2010. "Mujeres extranjeras en prisiones españolas. El caso andaluz". Revista Internacional de Sociología 68:473-498.

Cid, J. 2005. "The penitentiary system in Spain The use of imprisonment, living conditions and rehabilitation". Punishment \& Society 7:147-166. http://dx.doi.org/10.1177/1462474505050439

Cid, J. 2009. "Is imprisonment criminogenic? A comparative study of recidivism rates between prison and suspended prison sanctions". European Journal of Criminology 6:459-480. http://dx.doi.org/10.1177/1477370809341128

Cid, J. and J. Martí. 2012. "Turning points and returning points: Understanding the role of family ties in the process of desistance". European Journal of Criminology 9:603-620. http://dx.doi.org/10.1177/1477370812453102

Cobbina, J., B. Huebner and M. Berg. 2012. "Men, women and postrelease offending: an examination of the nature of the link between relational ties and recidivism". Crime and delinquency 58:331-361. http://dx.doi.org/10.1177/0011128710382348

Cohen, J. 1968. "Weighted Kappa: Nominal scale agreement with provision for scaled disagreement or partial credit". Psychologica Bulletin 70:213-220. http://dx.doi.org/10.1037/h0026256

Comfort, M. L. 2003. "In The Tube At San Quentin. The 'Secondary Prisonization' of Women Visiting Inmates". Journal of Contemporary Ethnography 32:77-107. http://dx.doi.org/10.1177/0891241602238939

Cullen, F. T. 1994. "Social Support as an Organizing Concept for Criminology: Presidential Address to the Academy of Criminal Justice Sciences". Justice Quarterly 11:527-559. http://dx.doi.org/10.1080/07418829400092421

Cullen, F. T. and J. Wright. 1997. "Liberating the anomie-strain paradigm: Implications from social-support theory". Pp. 187-206 in The future of anomie theory, edited by A. N. Passas and R. Agnew. Boston: Northeastern University Press.

Dixey, R. and J. Woodall. 2012. "The significance of the 'visit' in an English category-B prison: views form prisoners, prisoners' families and prison staff'. Community, Work \& Family 15:2947. http://dx.doi.org/10.1080/13668803.2011.580125

Duwe, G. and V. Clark. 2013. "Blessed be the social tie that binds: the effect on prison visitation on offender recidivism". Criminal Justice Policy Review 24:271-296. http://dx.doi.org/10.1177/0887403411429724 
Freedman, D., A. Thornton, D., Camburn D. D. Alwin and L. YoungdeMarco. 1988. "The life history calendar: a technique for collecting retrospective data". Sociological Methodology 18:37-68. http://dx.doi.org/10.2307/271044

García-Borés, J. 2003. "El impacto carcelario". Pp. 395-425 in Sistema penal y problemas sociales, editado por R. Bergalli. Valencia: Tirant lo Blanch.

García-Borés, P., N. Font, C. Fernández, R. Escurriol, A. Roig, H. Leyton y M. Moreno. 2006. La cárcel en el entorno familiar estudio de las repercusiones del encarcelamiento sobre las familias: problemáticas y necesidades. Barcelona: Ajuntament de Barcelona, Observatori del Sistema Penal i els Drets Humans-Universitat de Barcelona.

García-España E y J. L. Díez Ripollés, eds. 2012. Realidad y política penitenciarias. Valencia: Tirant lo Blanch.

Giordano, P. C., S. A. Cernkovich and L. Rudolph. 2002. "Gender, crime, and desistance:Toward a theory of cognitive transformation". American Journal of Sociology 107:990-1064. http://dx.doi.org/10.1086/343191

Giordano, P. C., S. A. Cernkovich and R. D. Schroeder. 2007. "Emotions and Crime over the Life Course: A Neo-Meadian Perspective on Criminal Continuity and Change". American Journal of Sociology 112:1603-1661. http://dx.doi.org/10.1086/512710

Glaser, D. 1964. The Effectiveness of a Prison and Parole System. Indianapolis, IN: Bobbs-Merrill.

Hairston, C. F. 1988. "Family ties during imprisonment: Do they influence future criminal activity?". Federal Probation 52:48-52.

La Vigne, N. G., C. Visher and J. Castro. 2004. Chicago prisoners' experiences returning home. Washington, DC: The Urban Institute.

La Vigne, N. G., R. L. Naser, L. E. Brooks and J. L. Castro. 2005. "Examining the Effect of Incarceration and In-Prison Family Contact on Prisoners' Family Relationships". Journal of Contemporary Criminal Justice 21:314-335. http://dx.doi.org/10.1177/1043986205281727

Laub, J. and R. Sampson. 2003. Shared beginnings, divergent lives: Delinquents boys to age 70. Cambridge, MA: Harvard University Press.

Lebel, T., R. Burnett, S. Maruna and S. Bushway. 2008. "The 'Chicken and Egg' of Subjective and Social Factors in Desistance". European Journal of Criminology 5:131-159. http://dx.doi.org/10.1177/1477370807087640

Lemert, E. 1967. Human Deviance, Social Problems and Social Control. Englewood Cliffs, NJ: Prentice Hall.

Lin, N. 1986. "Conceptualizing social support". Pp. 17-30 in Social Support, Life Events, and Depression, edited by N. Lin, A. Dean and W. M. Ensel. Orlando, FL: Academic Press.

Martínez, D. and L. S. Abrams. 2013. "Informal social support among returning young offenders: A metasynthesis of the literature". International Journal of Offender Therapy and Comparative Criminology 57:169-190. http://dx.doi.org/10.1177/0306624X11428203

Maruna, S. 2001. Making Good. How Ex-Convicts Reform and Rebuild their Lives. Washington, DC: American Psychological Association. http://dx.doi.org/10.1037/10430-000

Mears D., J. Cochran, S. Siennick and W. Bales. 2012. "Prison visitation and recidivism". Justice Quarterly 29:888-918. http://dx.doi.org/10.1080/07418825.2011.583932

Mills, A. and H. Codd. 2008. "Prisoners' families and offender management: mobilizing social capital". Probation Journal 55:9-24. http://dx.doi.org/10.1177/0264550507085675

Moreno, L. 2001. "La 'vía media' española del modelo de bienestar mediterráneo". Papers, Revista de Sociologia 63/64:67-82.

Ohlin, L. E. 1951. Selection for Parole. New York: Russell Sage.
Petersilia, J. 2003. When Prisoners come Home. Parole and Prisoner Reentry. Oxford: Oxford University Press.

Poehlmann, J., R. J. Shlafer, E. Maes and A. Hanneman. 2008. "Factors Associated With Young Children's Opportunities for Maintaining Family Relationships During Maternal Incarceration". Family Relations 57:267-280. http://dx.doi.org/10.1111/j.1741-3729.2008.00499.x

Rodríguez, J., M. Rovira and E. Larrauri. 2013. "The Effect of Having an Incarceration Record on Young Spanish Males' Odds of Finding Employment." Paper presented at The American Society of Criminology Annual Meeting, Nov 19, Chicago, IL.

Sampson, R. and J. Laub. 1993. Crime in the making: Pathways and turning points through life. Cambridge, MA: Harvard University Press.

Sampson, R., J. Laub and C. Wimer. 2006. "Does marriage reduce crime? A counterfactual approach to within-individual causal effects". Criminology 44:465-506. http://dx.doi.org/10.1111/j.1745-9125.2006.00055.x

Savolainen, J. 2009. "Work, Family and Criminal Desistance. Adult Social Bonds in a Nordic Welfare State". British Journal of Criminology 49:285-304. http://dx.doi.org/10.1093/bjc/azn084

Schroeder, R. D., P. C. Giordano and S. A. Cernkovich. 2010. Adult child-parent bonds and life course criminality. Journal of Criminal Justice 38:562-571. http://dx.doi.org/10.1016/j.jcrimjus.2010.04.027

Shover, N. 1985. Aging criminals. Beverly Hills: Sage.

Siennick, S. and D. Osgood. 2008. "A review of research on the impact on crime of transitions to adult roles". Pp. 161-187 in The long view of crime: A synthesis of longitudinal research, edited by A. M. Liberman. New York: Springer.

Small, M. L. 2011. "How to Conduct a Mixed Methods Study: Recent Trends in a Rapidly Growing Literature". Annual Review of Sociology 37:57-86. http://dx.doi.org/10.1146/annurev.soc.012809.102657

Visher, C. A. and D. O'Connell. 2012. "Incarceration and inmates' self perceptions about returning home". Journal of Criminal Justice 40:386-393. http://dx.doi.org/10.1016/j.jcrimjus.2012.06.007

Visher, C. A. and J. Travis. 2003. "Transitions from Prison to Community: Understanding Individual Pathways". Annual Review of Sociology 29:89-113. http://dx.doi.org/10.1146/annurev.soc.29.010202.095931

Vittinghoff, E. and C. E. McCulloch. 2007. "Relaxing the Rule of Ten Events per Variable in Logistic and Cox Regression". American Journal of Epidemiology 165:710-718. http://dx.doi.org/10.1093/aje/kwk052

Wakefield, S. and C. Uggen. 2004. "Having a Kid Changes Everything? The Effects of Parenthood on Subsequent Crime". Paper presented at the annual meeting of the American Sociological Association, August 18th, San Francisco, CA.

Wright, J. P., F. Cullen and J. Miller. 2001. "Family social capital and delinquent involvement". Journal of Criminal Justice 29:1-9. http://dx.doi.org/10.1016/S0047-2352(00)00071-4

JOEL MARTí es profesor de Sociología de la UAB y miembro del Centre d'Estudis Sociològics Sobre la Vida Quotidiana $i$ el Treball (QUIT), Institut d'Estudis del Treball (IET). Especializado en métodos y técnicas de investigación social.

JOSÉ CID es profesor de Derecho Penal de la UAB y coordinador del grupo de investigación Desistimento del delito y políticas de reinserción. Especializado en teoría criminológica, penología y desistimiento de la delincuencia. 